\title{
Industrial Patterns of Herding Beyond the Geographical Boundaries: An Empirical Investigation of Emerging Countries
}

\begin{abstract}
Anam Faraz Chaudry ${ }^{1}$, Ajid ur Rehman ${ }^{2}$, Muhammad Husnain ${ }^{3}$
Abstract

This study investigates the industrial pattern of "herding" in emerging countries including Pakistan, Philippine and Thailand. The uniqueness of the study is the utilization of a rich database from the three above mentioned countries with appropriate industrial classification. Return dispersion model including CSAD, CSSD linear model, non-linear regression and the bullish and bearish effects were applied on the daily returns of 262 companies from 6 sectors over a period of 2009 to 2016. The main findings of the study reveal that there are no signs of herding detected in the linear CSSD except in the energy sector of Pakistan. Moreover, in the linear CSAD model the only sign of herding was observed within the energy sector of Pakistani Stocks. The regression analysis revealed the presence of herding behavior in all 6 sectors of emerging countries except information technology sector. The study also provides evidence on the presence of herding behavior across bullish and bearish market conditions.
\end{abstract}

Keywords: Herding behavior, Industrial Herding, Return Dispersion Model, Emerging Markets

\section{JEL Classification: G14, G15}

\section{Introduction}

Psychology is the base of behavioral finance (Economou, Kostakis, \& Philippas, 2010). Psychological factors are the key drivers of irrationality (Simon's, 1955). Investors avoid complex procedures when they face uncertain situations and this avoidance escalates biases in investment decisions and resultantly, investors deviate from rationality. Herding behavior is one of the most common behavioral biases of investors. Balcilar, Demirer, and Hammoudeh (2013) defined herding as investor ignoring

1 MS-Scholar, Faculty of Management Sciences, Riphah International University, Islamabad. Email:anamfaraz@live.com

2 Assistant Professor, Faculty of Management Sciences, Riphah International University, Islamabad.

Email: ajid.rehman@riphah.edu.pk

3 Assistant Professor, Faculty of Management Sciences, Riphah International University, Lahore.

\begin{tabular}{ll} 
ARTICLE HISTORY & \\
26 July, 2018 Submission Received & 06 Nov, 2018 First Review \\
\hline 15 Mar, 2019 Second Review & 29 Sep, 2019 Third Review \\
\hline
\end{tabular}

15 Oct, 2019 Accepted 
personal beliefs and evaluation process and walks behind other investors. Through the neuroeconomic perspective, Prechter (2001) predicts herding as an emotional mental activity influenced by the behavior of others that deviate from rationality in situations. (Spyrou, 2013).

Companies in one single industry are likely to be affected by similar economic conditions. Therefore, herding is expected in given industry rather than the whole market. The pattern of growth is same in industries across the emerging countries due to the competitiveness (Zheng, Li \& Chiang, 2017). There are also number of evidences from developed and emerging markets that prove the existence of sectoral herding. For example, studies of Demirer, Kutan, \& Zhang (2014), Yao, Ma, \& He (2014), Litimi, BenSaida, \& Bouraoui (2016) in US industries, Dehghani \& Sapian (2014) in Malaysian industries and Zheng, Li, \& Chiang (2017) in Asian markets.

Firstly companies selected in our sample are included in the top 20 emerging markets which have shown significant growth since last many years i.e., Indonesia, Philippines, and Thailand with 31.3\%, 20.4\%, and 25.9\% growth rate respectively (Bloomberg, 2013). Secondly, the strand of herding behavior across industrial sectors lacks an extended investigation. The investigation across the sectorial level will greatly add value regarding the investment decisions of investors specially for those investors who follow the herding strategy in their investments. Thirdly, previous studies on emerging markets which have focused on the Asian markets have included limited sample for their investigation. Most of the studies focused on the whole markets rather than investigating herding behavior across industries (Jhandir \& Elahi 2014; Javed, Zafar, \& Hafeez, 2011; Shah, Shah and Khan, 2017). Our study investigates the phenomena across an extended dataset of 262 companies with industry classification according to the Global Industry Classification Codes. From the different market conditions context, the study adds to the existing body of knowledge regarding herding behavior by incorporating different market conditions in line with Youssef and Mokni (2018).

Further the study contributes to the investor decision making processes, especially, the investigation across the sectorial level will greatly add value regarding the investment decisions of investors specially for those investors who follow the herding strategy in their investments

The paper organized as follows. First the literature review then research methodology that is followed by the data analysis and discussion of findings and finally the conclusion of research along with the future directions and study implications.

4 https://www.bloomberg.com/news/photo-essays/2013-01-31/the-top-20-emerging-markets 


\section{Literature Review}

\subsection{Herding behavior}

The financial terminology "Herding behavior" is a behavior of an investor to copy trading activities of other investors (Bikhchandani \& Sharma, 2001). Usually investors follow trends. Christie \& Huang, (1995) and Patterson \& Sharma, (2007) noted that "...herding occurs when a group of investors trade on the same side of the market in the same securities over the same period or when investors ignore their private information and act what other investors do". Herding behavior can upset the stability of financial markets (Dhar \& Zhu, 2006) as a result of this instability stock prices deviate from their fundamental values (Patterson \& Sharma, 2007) so information plays a vital role in herding behavior (Avery \& Zemsky, 1998, Cipriani \& Guarino, 2008). Balciler et al., (2013) defined herding as Investor ignores personal beliefs and evaluation process and walks behind other investors. Through the neuroeconomic perspective, Prechter (2001) predicted herding as an emotional mental activity influenced by the behavior of others that deviate from rationality in situations (Spyrou, 2013).

Herding behavior is frequently associated with the volatility of stock returns and mostly herding behavior observe in extreme market movements (Christie \& Huang, 1995). Emerging markets are more volatile than the developed markets (Peranginangin, Ali, Brockman, \& Zurbruegg, 2016). After the crisis of 1997 emerging markets have employed numerous measures for the sophistication and development of their financial system. As a result, investor interest has greatly increased across these markets. According to global market classification across the strands of developed and emerging markets, investor usually prefers to invest in emerging markets because emerging markets have new investment avenues that provide both higher returns and rapid growth. For example, the study of Hsieh, Yang, Yang and Lee (2011) found herding in several emerging markets, Balcilar et al. (2013) in GulfArab, Demirer, Kutan, and Chen, (2010) and Bowe and Domuta (2004) in Jakarta Stock Exchange. All these examples provide the evidence of herding in frontier/emerging markets.

\subsection{Empirical evidence from industrial herding}

Globally Herding behavior is now tested at industrial level as compared to domestic or international level. The empirical investigation of Demirer, Kutan and Zhang (2014) and Yao et al. (2014) provide empirical evidence that herding behavior is extensive in industry rather than overall market. Another study of Litimi et al. (2016) took US industries as a study sample and observed herding in consumer non-durables, energy, health care, public utilities, technology, and transportation. 
Industries such as materials, consumer services, and oil and gas possess more herding then other industries (Gebka \& Wohar 2013). The industrial based study conducted in Malaysian industries by Dehghani and Sapian (2014) reveals that herding behavior is only observed in technology sector. Lee, Chen \& Hsieh (2013) and Yao et al. (2014) also evaluate the industrial herding in Chinese stock market. In Asian markets specifically Japan, China, South Korea, Hong Kong, Taiwan, Singapore, Indonesia, Malaysia, and Thailand, when asset pricing model and cross-sectional dispersion of stock returns models are applied, it was revealed that herding is stronger at industry level as compared to domestic and international market levels. Herding behavior are stronger in the technology and financial industries but weaker in the utility industry (Zheng et al., 2017).

\subsection{Empirical evidence from developed markets}

Develop markets are the cream of total market. Markets that have most developed capital markets, also exhibit herding behavior. Chiang and Zheng (2010) investigated Latin American and Asian markets, Australia, France, Germany, Hong Kong, Japan, United Kingdom, United States, Argentina, Brazil, Chile, Mexico, China, Indonesia, Malaysia, Singapore, South Korea, Taiwan, and Thailand. They reported evidence of herding in bull and bearish trend and asymmetric herding in advance markets except United States, and reported no evidence of herding in Latin America. Kremer and Nautz (2013) find evidence of herding in Germany. The study of Asma, Mollah, and Keasey (2014) found herding in European countries during European crisis. There are number of studies available that prove existence of herding behavior in develop markets like Economou, Kostakis, and Philippas (2011) and Philippas, Economou, Babalos and Kostakis (2013).

\subsection{Empirical evidence from frontier/emerging markets}

Frontier/emerging markets that are one step behind the developed markets. Balc1lar, Demirer \& Ulussever (2017) investigated the speculation in the oil market that drive investor herding in frontier stock markets. They took a sample of 4 countries including Saudi Arabia, United Arab Emirates, Kuwait and Qatar and applied return dispersion models. The study results show significant signs of herding in emerging countries and during high volatility period. Shah et al. (2017) reported the presence of herding behavior in stock exchange of Pakistan. A sample of 261 companies of Pakistan was studied by Malik and Elahi (2014) and found significant signs of herding in Pakistan. Emerging markets including China exhibit herding behavior (Hsieh, Yang, Yang, \& Lee 2011, Demirer et al., 2010). The results of Balcilar et al., (2013) suggest that herding exist in GulfArab stock markets (Abu Dhabi, Dubai, Kuwait, Qatar and Saudi Arabia). There results also suggest that the frontier markets have different in 
structure than developed markets. Lakshman, Basu, and Vaidyanathan, (2013) studied herding behavior in India and came up with that efficient Indian markets does not possess herding. In most of the cases herding is observed in crisis periods like the study of Bowe and Domuta (2004) found significant herding in Jakarta Stock Exchange during Asian financial crisis in 1997. Javed et al. (2011) reported herding behavior in the Pakistan, KSE-100 Index. Evidence of herding from 8 African stock exchanges by using Cross section dispersion models were confirmed in Botswana, Ghana, Kenya, Namibia, Nigeria, Tanzania and Zambia, a study conducted by Guney, Kallinterakis, Komba (2016)

\section{Methodology}

\subsection{Data and data sources}

The study is based on the emerging markets. According to Morgan Stanley Capital International

(MSCI Inc). market classification 2017 Pakistan Philippines and Thailand are included in emerging markets. The data used in this research consists of daily returns of the companies that belong to emerging markets from 2009-2016. The division of industries and companies within the industries is based on Global Industry Classification Standard (GICS). Table 1 contains the information about industries and the number of companies in each industry. The sample of study consists of 262 companies and historical data is obtained from Bloomberg data base.

Table 1: Companies/Industries Information

\begin{tabular}{|c|c|c|c|}
\hline Industries /Countries & Pakistan & Philippines & Thailand \\
\hline Consumer Discretionary & 12 & 16 & 8 \\
\hline Energy & 9 & 8 & 7 \\
\hline Financials & 23 & 64 & 23 \\
\hline Industrials & 5 & 13 & 12 \\
\hline Information Technology & $\mathrm{n} / \mathrm{a}$ & 13 & 6 \\
\hline Materials & 18 & 19 & 6 \\
\hline Total number of companies & 67 & 133 & 62 \\
\hline
\end{tabular}

Table 1 present the number companies in each industry. 


\subsection{Return dispersion models}

The study employs return dispersion models in order to investigate the herding behavior across the industries. The return dispersion models based on cross-sectional standard deviations (CSSD) and cross-sectional absolute standard deviations (CSAD) are employed to detect the herding behavior among the investors of emerging countries. Christie and Huang (1995), Vo and Phan (2017) used CSSD and Chang, Cheng, and Khorana (2000) used CSAD to measure the average proximity of individual asset returns to the realized industry average and non-linear regression to examine the relation between the level of equity returns dispersions and the overall industry returns respectively. Before using the methodologies, some information is extracted from the available data. The mathematical expression of stock return is

$$
R_{c, t}=\operatorname{Ln}\left(\frac{P_{t}}{P_{t-1}}\right)
$$

Where $\mathrm{R}_{c, t}$ is the stock return of company in industry at time t. $\mathrm{P}_{t}$ and $\mathrm{P}_{\mathrm{t}-1}$ are the closing price of the stock at time $t$ and $t-1$. The average stock returns are calculated by using formula:

$$
R_{j, t}=\frac{\sum R_{c, t}}{N}
$$

Where $\mathrm{R}_{\mathrm{i}, \mathrm{t}}$ is the average stock returns of $\mathrm{N}$ numbers of companies in industry at time $t$.

The return dispersion is measured by CSSD, the methodology already used by Christie and Huang (1995), Chang et al. (2000), Gleason, Lee, and Mathur (2003), Lin and Swanson (2003), Gleason Gleason, Mathur, and Peterson (2004), and Demirer and Kutan (2006). The mathematical expression of Cross-sectional standard deviations (CSSD) is:

$$
\operatorname{CSSD}_{t}=\sqrt{\frac{\sum_{i=1}^{N}\left(R_{c, t}-R_{j, t}\right)^{2}}{N-1}}
$$

Where $R_{c, t}$ is the stock return of company at time $t, R_{j, t}$ is the average return of $\mathrm{N}$ (number of companies) in a particular industry at time t.

The methodology is based on the idea that if there is herding in industry then the security returns will deviate from the overall industry return. The assumption behind this idea is simple that investor make decisions in accordance with other investors, but rational asset pricing models suggest that dispersion increases with the absolute market return. CSSD $_{t}$ also suggests that in extreme market movements herding is most likely to occur. In the context of extreme market conditions, we follow Youssef and Mokni (2018) and Chaffai and Medhioub (2018) by dividing the returns into an upper tail and lower tail of returns. Thus, to test herding following regression model 
has been used:

$$
\operatorname{CSSD}_{t}=\alpha+\beta_{1}^{U} D_{t}^{U}+\beta_{2}^{L} D_{t}^{L}+\varepsilon_{t}
$$

Where $\mathrm{D}_{t}^{\mathrm{U}}$ and $\mathrm{D}_{t}^{\mathrm{L}}$ are the dummies for $\beta_{1}$ and $\beta_{2}$. The indication of herd formation is based on the values of beta. When $\beta_{1}$ and $\beta_{2}$ coefficients are negative and statistical significant, then herding behavior exist among the investors and if $\beta_{1}$ and $\beta_{2}$ coefficients are significantly positive then they hold rational asset pricing model.

The second methodology of return dispersion models is based on cross-sectional absolute standard deviations (CSAD). CSAD was proposed by Chang et al. (2000). The mathematical expression of CSAD is:

$$
C S A D_{t}=\frac{1}{N} \sum_{i=1}^{N}\left|R_{c, t}-R_{j, t}\right|
$$

Where $R_{c, t}$ is the stock return of company at time $t, R_{j, t}$ is the average return of $\mathrm{N}$ (number of companies) in a particular industry at time $t$. In this methodology this study confronts the capital asset pricing model assumption that there is a linear relationship among market dispersion and market return. The study proposed non-linear relationship among market dispersion and market return and proposed a test methodology that is:

$$
C S A D_{t}=\alpha+\gamma_{1}\left|R_{j t}\right|+\gamma_{2} R_{j t}^{2}+\varepsilon_{t}
$$

Where $\mathrm{R}_{\mathrm{it}}$ is the average return of $\mathrm{N}$ (number of companies) in a industry at time t. The indication of herd formation as evidenced by lower proportional increase in CSAD during extreme market movements and will be negative and statistically significant. If is statistically significant but positive, then there will be no evidence of herd formation. This non-linear relationship was criticized by Gleason et al. (2004) and he argued this non-linear relationship can also be used for CSSD therefore Gleason et al. (2004) employ two additional test by interchanging the dependent variables in equation 4 and 6 :

$$
\begin{aligned}
& C S A D_{t}=\alpha+\beta_{1}^{U} D_{t}^{U}+\beta_{2}^{L} D_{t}^{L}+\varepsilon_{t} \\
& C S S D_{t}=\alpha+\gamma_{1}\left|R_{j t}\right|+\gamma_{2} R_{j t}^{2}+\varepsilon_{t}
\end{aligned}
$$

Where $\mathrm{R}_{\mathrm{it}}$ is the average return of $\mathrm{N}$ (number of companies) in a particular industry. The indication of herd formation is evidenced by lower proportional increase in CSSD during extreme market movements and will be negative and statistically significant. If is statistically significant but positive, then there will be evidence of herd formation. As there are two main market trends bullish and bearish. The rate of increase in dispersion with respect to aggregate market return is higher in bullish 
trend and lower in bearish trend. To observe the asymmetry in bullish and bearish trends the herding regression is estimated separately for positive industry returns and negative industry returns. The mathematical expressions are:

$$
C S A D_{t}^{U P}=\alpha+\gamma_{1}^{U P}\left|R_{j, t}^{U P}\right|+\gamma_{2}^{U P}\left(R_{j, t}^{U P}\right)^{2}+\varepsilon_{t} \text { if } R_{j, t}>0
$$

Where $\mathrm{R}^{\mathrm{UP}}{ }_{\mathrm{j}, \mathrm{t}}$ is the average return of $\mathrm{N}$ (number of companies). The indication of herd formation is evidenced when $\gamma_{2}^{U P}$ will be negative and statistically sgnificant. If $\gamma^{U P}{ }_{2}$ is statistically significant but positive, then there is no evidence of herd formation.

$$
C S A D_{t}^{\text {DOWN }}=\alpha+\gamma_{1}^{\text {DOWN }}\left|R_{j, t}^{\text {DOWN }}\right|+\gamma_{2}^{\text {DOWN }}\left(R_{j, t}^{\text {DOWN }}\right)^{2}+\varepsilon_{t} \text {, if } R_{j, t}<0
$$

Where $\mathrm{R}^{\mathrm{DOWN}}$ i,t is the average return of $\mathrm{N}$ (number of companies). The indication of herd formation is evidenced when $\gamma^{\text {DOWN }}{ }_{2}$ will be negative and statistically significant. If $\gamma_{2}^{U P}$ is statistically significant but positive, then there is no evidence of herd formation and if any coefficient is statistically insignificant then there is no sign of herding. The autocorrelation and heteroscedasticity in data is controlled by using Newey West consistent estimator (1987) test.

\subsection{Findings and discussion}

\subsection{Descriptive statistic}

Table 2: Descriptive Statistic

\begin{tabular}{|c|c|c|c|c|c|c|}
\hline Countries & \multicolumn{2}{|c|}{ Pakistan } & \multicolumn{2}{c|}{ Philippines } & \multicolumn{2}{c|}{ Thailand } \\
\hline Sectors & Mean & SD & Mean & SD & Mean & SD \\
\hline $\begin{array}{c}\text { Consumer } \\
\text { Discretionary }\end{array}$ & 0.053 & 1.238 & 0.005 & 1.086 & 0.050 & 1.384 \\
\hline Energy & 0.016 & 1.379 & -0.001 & 1.487 & 0.004 & 1.665 \\
\hline Financials & -0.001 & 1.348 & 0.006 & 1.011 & 0.033 & 1.598 \\
\hline Industrials & 0.131 & 1.454 & 0.034 & 1.248 & 0.024 & 1.710 \\
\hline $\begin{array}{c}\text { Information } \\
\text { Technology }\end{array}$ & - & - & 0.040 & 1.365 & 0.059 & 1.441 \\
\hline Materials & 0.044 & 1.468 & -0.032 & 1.648 & 0.050 & 1.480 \\
\hline
\end{tabular}

Table 2 present the descriptive statistic of each sector.

The results of descriptive statistic (table 2) show both returns and volatility. In all sectors we observe both positive and negative average daily returns. Pakistan has highest average daily returns in consumer discretionary, energy, and industrials. Thailand has highest daily average returns in material. If we discuss about the highest average 
volatility, Thailand have highest average volatility in consumer discretionary, financial, and industrials. Philippine has highest average volatility in materials.

Table 3: Regression Coefficients for Linear Herding Models

\begin{tabular}{|c|c|c|c|c|c|c|}
\hline \multicolumn{7}{|c|}{ Consumer Discretionary } \\
\hline \multicolumn{7}{|c|}{ Panel A: Linear herding estimates for CSSD on market portfolio } \\
\hline & & $\begin{array}{c}\text { Using } 1 \% \\
\text { criterion }\end{array}$ & & & $\begin{array}{l}\text { Using 5\% } \\
\text { criterion }\end{array}$ & \\
\hline Countries & A & $\beta_{\mathrm{L}}$ & $\beta^{\cup}$ & A & $\beta_{\mathrm{L}}$ & $\beta^{\cup}$ \\
\hline Pakistan & 0.027 & $0.014^{* * *}$ & $0.012^{* * *}$ & 0.021 & $0.010^{* * *}$ & $0.010^{* * *}$ \\
\hline Philippine & 0.027 & $0.048^{* * *}$ & $0.058^{* * *}$ & 0.025 & $0.020^{* * *}$ & $0.035^{* * *}$ \\
\hline \multirow[t]{2}{*}{ Thailand } & 0.017 & $0.020^{* * *}$ & $0.022^{* * *}$ & 0.016 & $0.010^{* * *}$ & $0.014^{* * *}$ \\
\hline & & $\begin{array}{l}\text { Using } 10 \% \\
\text { criterion }\end{array}$ & & & $\begin{array}{l}\text { Using 15\% } \\
\text { criterion }\end{array}$ & \\
\hline Countries & A & $\beta_{\mathrm{L}}$ & $\beta^{\cup}$ & A & $\beta_{\mathrm{L}}$ & $\beta^{\mathrm{U}}$ \\
\hline Pakistan & 0.021 & $0.007^{* * *}$ & $0.007^{* * *}$ & 0.020 & $0.006^{* * *}$ & $0.006^{* * *}$ \\
\hline Philippine & 0.024 & $0.017^{* * *}$ & $0.025^{* * *}$ & 0.023 & $0.013^{* * *}$ & $0.021^{* * *}$ \\
\hline Thailand & 0.016 & $0.007^{* * *}$ & $0.010^{* * *}$ & 0.015 & $0.006^{* * *}$ & $0.010^{* * *}$ \\
\hline \multicolumn{7}{|c|}{ Panel B: Linear herding estimates for CSAD on market portfolio } \\
\hline & & $\begin{array}{c}\text { Using } 1 \% \\
\text { criterion }\end{array}$ & & & $\begin{array}{l}\text { Using 5\% } \\
\text { criterion }\end{array}$ & \\
\hline Countries & A & $\beta_{\mathrm{L}}$ & $\beta^{\cup}$ & A & $\beta_{\mathrm{L}}$ & $\beta^{\cup}$ \\
\hline Pakistan & 0.016 & $0.009^{* * *}$ & $0.008^{* * *}$ & 0.016 & $0.009^{* * *}$ & $0.008^{* * *}$ \\
\hline Philippine & 0.017 & $0.029^{* * *}$ & $0.032^{* * *}$ & 0.016 & $0.014^{* * *}$ & $0.021^{* * *}$ \\
\hline \multirow[t]{2}{*}{ Thailand } & 0.013 & $0.015^{* * *}$ & $0.017^{* * *}$ & 0.012 & $0.008^{* * *}$ & $0.011^{* * *}$ \\
\hline & & $\begin{array}{c}\text { Using 10\% } \\
\text { criterion }\end{array}$ & & & $\begin{array}{l}\text { Using 15\% } \\
\text { criterion }\end{array}$ & \\
\hline Countries & A & $\beta_{\mathrm{L}}$ & $\beta^{\cup}$ & A & $\beta_{\mathrm{L}}$ & $\beta^{\mathrm{U}}$ \\
\hline Pakistan & 0.015 & $0.007^{* * *}$ & $0.007^{* * *}$ & 0.015 & $0.006^{* * *}$ & $0.006^{* * *}$ \\
\hline Philippine & 0.015 & $0.012^{* * *}$ & $0.016^{* * *}$ & 0.014 & $0.010^{* * *}$ & $0.014^{* * *}$ \\
\hline Thailand & 0.012 & $0.005^{* * *}$ & $0.008^{* * *}$ & 0.011 & $0.005^{* * *}$ & $0.007^{* * *}$ \\
\hline \multicolumn{7}{|c|}{ Energy } \\
\hline & Panel A & near herding & timates for & D on ma & portfolio & \\
\hline
\end{tabular}




\begin{tabular}{|c|c|c|c|c|c|c|}
\hline & & $\begin{array}{l}\text { Using 1\% } \\
\text { criterion }\end{array}$ & & & $\begin{array}{l}\text { Using 5\% } \\
\text { criterion }\end{array}$ & \\
\hline Countries & A & $\beta_{\mathrm{L}}$ & $\beta^{\mathrm{U}}$ & $\mathrm{A}$ & $\beta_{\mathrm{L}}$ & $\beta^{\mathrm{U}}$ \\
\hline Pakistan & $0.004^{* * *}$ & 0.001 & $-0.002^{* *}$ & $0.004^{* * *}$ & $0.003^{* * *}$ & 0.001 \\
\hline Philippine & $0.022^{* * *}$ & $0.036^{* * *}$ & $0.039^{* * *}$ & $0.021^{* * *}$ & $0.019^{* * *}$ & $0.023^{* * *}$ \\
\hline \multirow[t]{2}{*}{ Thailand } & $0.013^{* * *}$ & $0.022^{* * *}$ & $0.048^{* * *}$ & $0.013^{* * *}$ & $0.011^{* * *}$ & $0.019^{* * *}$ \\
\hline & & $\begin{array}{c}\text { Using } 10 \% \\
\text { criterion }\end{array}$ & & & $\begin{array}{c}\text { Using } 15 \% \\
\text { criterion }\end{array}$ & \\
\hline Countries & A & $\beta_{\mathrm{L}}$ & $\beta^{\mathrm{U}}$ & A & $\beta_{L}$ & $\beta^{\mathrm{U}}$ \\
\hline Pakistan & 0.004 & $0.002^{* * *}$ & $0.001^{* * *}$ & 0.003 & $0.002^{* * *}$ & $0.002^{* * *}$ \\
\hline Philippine & 0.019 & $0.014^{* * *}$ & $0.020^{* * *}$ & 0.018 & $0.013^{* * *}$ & $0.016^{* * *}$ \\
\hline Thailand & 0.012 & $0.008^{* * *}$ & $0.013^{* * *}$ & 0.012 & $0.006^{* * *}$ & $0.011^{* * *}$ \\
\hline \multicolumn{7}{|c|}{ Panel B: Linear herding estimates for CSAD on market portfolio } \\
\hline & & $\begin{array}{l}\text { Using 1\% } \\
\text { criterion }\end{array}$ & & & $\begin{array}{l}\text { Using 5\% } \\
\text { criterion }\end{array}$ & \\
\hline Countries & A & $\beta_{\mathrm{L}}$ & $\beta^{\mathrm{U}}$ & A & $\beta_{\mathrm{L}}$ & $\beta^{\mathrm{U}}$ \\
\hline Pakistan & $0.009^{* * *}$ & 0.002 & $-0.003^{* *}$ & $0.009^{* * *}$ & $0.007^{* * *}$ & $0.004^{* * *}$ \\
\hline Philippine & $0.017^{* * *}$ & $0.032^{* * *}$ & $0.035^{* * *}$ & $0.015^{* * *}$ & $0.018^{* * *}$ & $0.021^{* * *}$ \\
\hline \multirow[t]{2}{*}{ Thailand } & $0.01^{* * *}$ & $0.016^{* * *}$ & $0.033^{* * *}$ & $0.009^{* * *}$ & $0.009^{* * *}$ & $0.014^{* * *}$ \\
\hline & & $\begin{array}{c}\text { Using } 10 \% \\
\text { criterion }\end{array}$ & & & $\begin{array}{c}\text { Using } 15 \% \\
\text { criterion }\end{array}$ & \\
\hline Countries & A & $\beta_{\mathrm{L}}$ & $\beta^{\mathrm{U}}$ & A & $\beta_{\mathrm{L}}$ & $\beta^{\mathrm{U}}$ \\
\hline Pakistan & 0.008 & $0.005^{* * *}$ & $0.004^{* * *}$ & 0.008 & $0.005^{* * *}$ & $0.004^{* * *}$ \\
\hline Philippine & 0.014 & $0.014^{* * *}$ & $0.018^{* * *}$ & 0.013 & $0.012^{* * *}$ & $0.015^{* * *}$ \\
\hline Thailand & 0.009 & $0.006^{* * *}$ & $0.010^{* * *}$ & 0.009 & $0.005^{* * *}$ & $0.008^{* * *}$ \\
\hline \multicolumn{7}{|c|}{ Financials } \\
\hline \multicolumn{7}{|c|}{ Panel A: Linear herding estimates for CSSD on market portfolio } \\
\hline & & $\begin{array}{l}\text { Using 1\% } \\
\text { criterion }\end{array}$ & & & $\begin{array}{l}\text { Using 5\% } \\
\text { criterion }\end{array}$ & \\
\hline Countries & A & $\beta_{\mathrm{L}}$ & $\beta^{\mathrm{U}}$ & A & $\beta_{\mathrm{L}}$ & $\beta^{\mathrm{U}}$ \\
\hline Pakistan & $0.018^{* * *}$ & $0.011^{* * *}$ & $0.004^{* *}$ & $0.017^{* * *}$ & $0.009^{* * *}$ & $0.008^{* * *}$ \\
\hline Philippine & $0.026^{* * *}$ & $0.026^{* * *}$ & $0.026^{* * *}$ & $0.024^{* * *}$ & $0.014^{* * *}$ & $0.022^{* * *}$ \\
\hline \multirow[t]{2}{*}{ Thailand } & $0.018^{* * *}$ & $0.021^{* * *}$ & $0.019^{* * *}$ & $0.017^{* * *}$ & $0.012^{* * *}$ & $0.013^{* * *}$ \\
\hline & & $\begin{array}{c}\text { Using } 10 \% \\
\text { criterion }\end{array}$ & & & $\begin{array}{c}\text { Using } 15 \% \\
\text { criterion }\end{array}$ & \\
\hline Countries & A & $\beta_{\mathrm{L}}$ & $\beta^{\mathrm{U}}$ & $\mathrm{A}$ & $\beta_{\mathrm{L}}$ & $\beta^{\mathrm{U}}$ \\
\hline
\end{tabular}




\begin{tabular}{|c|c|c|c|c|c|c|}
\hline Pakistan & 0.017 & $0.009^{* * *}$ & $0.008^{* * *}$ & 0.016 & $0.007^{* * *}$ & $0.007^{* * *}$ \\
\hline Philippine & 0.023 & $0.012^{* * *}$ & $0.018^{* * *}$ & 0.022 & $0.010^{* * *}$ & $0.016^{* * *}$ \\
\hline Thailand & 0.017 & $0.008^{* * *}$ & $0.011^{* * *}$ & 0.016 & $0.006^{* * *}$ & $0.009^{* * *}$ \\
\hline \multicolumn{7}{|c|}{ Panel B: Linear herding estimates for CSAD on market portfolio } \\
\hline & & $\begin{array}{l}\text { Using 1\% } \\
\text { criterion }\end{array}$ & & & $\begin{array}{c}\text { Using 5\% } \\
\text { criterion }\end{array}$ & \\
\hline Countries & A & $\beta_{\mathrm{L}}$ & $\beta^{\mathrm{U}}$ & A & $\beta_{L}$ & $\beta^{\mathrm{U}}$ \\
\hline Pakistan & $0.013^{* * *}$ & $0.006^{* * *}$ & 0.002 & $0.013^{* * *}$ & $0.008^{* * *}$ & $0.006^{* * *}$ \\
\hline Philippine & $0.016^{* * *}$ & $0.022^{* * *}$ & $0.02^{* * *}$ & $0.015^{* * *}$ & $0.012^{* * *}$ & $0.014^{* * *}$ \\
\hline \multirow[t]{2}{*}{ Thailand } & $0.013^{* * *}$ & $0.016^{* * *}$ & $0.015^{* * *}$ & $0.012^{* * *}$ & $0.009^{* * *}$ & $0.01^{* * *}$ \\
\hline & & $\begin{array}{l}\text { Using } 10 \% \\
\text { criterion }\end{array}$ & & & $\begin{array}{l}\text { Using } 15 \% \\
\text { criterion }\end{array}$ & \\
\hline Countries & A & $\beta_{\mathrm{L}}$ & $\beta^{\mathrm{U}}$ & A & $\beta_{\mathrm{L}}$ & $\beta^{\mathrm{U}}$ \\
\hline Pakistan & 0.012 & $0.007^{* * *}$ & $0.007^{* * *}$ & 0.012 & $0.006^{* * *}$ & $0.006^{* * *}$ \\
\hline Philippine & 0.014 & $0.009^{* * *}$ & $0.011^{* * *}$ & 0.013 & $0.008^{* * *}$ & $0.010^{* * *}$ \\
\hline Thailand & 0.012 & $0.006^{* * *}$ & $0.008^{* * *}$ & 0.012 & $0.005^{* * *}$ & $0.007^{* * *}$ \\
\hline \multicolumn{7}{|c|}{ Industrials } \\
\hline \multicolumn{7}{|c|}{ Panel A: Linear herding estimates for CSSD on market portfolio } \\
\hline & & $\begin{array}{l}\text { Using 1\% } \\
\text { criterion }\end{array}$ & & & $\begin{array}{c}\text { Using 5\% } \\
\text { criterion }\end{array}$ & \\
\hline Countries & A & $\beta_{\mathrm{L}}$ & $\beta^{\mathrm{U}}$ & A & $\beta_{L}$ & $\beta^{\mathrm{U}}$ \\
\hline Pakistan & $0.018^{* * *}$ & -0.005 & 0.003 & $0.017^{* * *}$ & $0.008^{* *}$ & 0.004 \\
\hline Philippine & $0.019^{* * *}$ & $0.022^{* * *}$ & $0.029^{* * *}$ & $0.018^{* * *}$ & $0.014^{* * *}$ & $0.02^{* * *}$ \\
\hline \multirow[t]{2}{*}{ Thailand } & $0.018^{* * *}$ & $0.023^{* * *}$ & $0.03^{* * *}$ & $0.017^{* * *}$ & $0.013^{* * *}$ & $0.019^{* * *}$ \\
\hline & & $\begin{array}{c}\text { Using } 10 \% \\
\text { criterion }\end{array}$ & & & $\begin{array}{c}\text { Using } 15 \% \\
\text { criterion }\end{array}$ & \\
\hline Countries & A & $\beta_{\mathrm{L}}$ & $\beta^{\mathrm{U}}$ & A & $\beta_{\mathrm{L}}$ & $\beta^{\mathrm{U}}$ \\
\hline Pakistan & 0.017 & $0.006^{* * *}$ & $0.004^{* * *}$ & 0.016 & $0.004^{* * *}$ & $0.005^{* * *}$ \\
\hline Philippine & 0.017 & $0.010^{* * *}$ & $0.015^{* * *}$ & 0.017 & $0.009^{* * *}$ & $0.013^{* * *}$ \\
\hline Thailand & 0.017 & $0.009^{* * *}$ & $0.016^{* * *}$ & 0.016 & $0.007^{* * *}$ & $0.013^{* * *}$ \\
\hline \multicolumn{7}{|c|}{ Panel B: Linear herding estimates for CSAD on market portfolio } \\
\hline & & $\begin{array}{l}\text { Using 1\% } \\
\text { criterion }\end{array}$ & & & $\begin{array}{c}\text { Using 5\% } \\
\text { criterion }\end{array}$ & \\
\hline Countries & A & $\beta_{\mathrm{L}}$ & $\beta^{\mathrm{U}}$ & A & $\beta_{\mathrm{L}}$ & $\beta^{\mathrm{U}}$ \\
\hline Pakistan & $0.013^{* * *}$ & -0.002 & 0.005 & $0.013^{* * *}$ & $0.008^{* * *}$ & $0.004^{*}$ \\
\hline
\end{tabular}




\begin{tabular}{|c|c|c|c|c|c|c|}
\hline Philippine & $0.014^{* * *}$ & $0.018^{* * *}$ & $0.022^{* * *}$ & $0.013^{* * *}$ & $0.011^{* * *}$ & $0.014^{* * *}$ \\
\hline \multirow[t]{2}{*}{ Thailand } & $0.014^{* * *}$ & $0.019^{* * *}$ & $0.023^{* * *}$ & $0.013^{* * *}$ & $0.011^{* * *}$ & $0.015^{* * *}$ \\
\hline & & $\begin{array}{c}\text { Using 10\% } \\
\text { criterion }\end{array}$ & & & $\begin{array}{l}\text { Using 15\% } \\
\text { criterion }\end{array}$ & \\
\hline Countries & A & $\beta_{\mathrm{L}}$ & $\beta^{\mathrm{U}}$ & A & $\beta_{\mathrm{L}}$ & $\beta^{\mathrm{U}}$ \\
\hline Pakistan & 0.012 & $0.006^{* * *}$ & $0.004^{* * *}$ & 0.012 & $0.004^{* * *}$ & $0.005^{* * *}$ \\
\hline Philippine & 0.012 & $0.009^{* * *}$ & $0.011^{* * *}$ & 0.012 & $0.007^{* * *}$ & $0.010^{* * *}$ \\
\hline Thailand & 0.012 & $0.008^{* * *}$ & $0.012^{* * *}$ & 0.012 & $0.006^{* * *}$ & $0.010^{* * *}$ \\
\hline \multicolumn{7}{|c|}{ Information Technology } \\
\hline \multicolumn{7}{|c|}{ Panel A: Linear herding estimates for CSSD on market portfolio } \\
\hline & & $\begin{array}{l}\text { Using 1\% } \\
\text { criterion }\end{array}$ & & & $\begin{array}{l}\text { Using 5\% } \\
\text { criterion }\end{array}$ & \\
\hline Countries & A & $\beta_{\mathrm{L}}$ & $\beta^{\mathrm{U}}$ & A & $\beta_{\mathrm{L}}$ & $\beta^{\mathrm{U}}$ \\
\hline Pakistan & $\mathrm{n} / \mathrm{a}$ & $\mathrm{n} / \mathrm{a}$ & $\mathrm{n} / \mathrm{a}$ & $\mathrm{n} / \mathrm{a}$ & $\mathrm{n} / \mathrm{a}$ & $\mathrm{n} / \mathrm{a}$ \\
\hline Philippine & $0.035^{* * *}$ & 0.011 & $0.059^{* * *}$ & $0.031^{* * *}$ & $0.019^{* *}$ & $0.065^{* * *}$ \\
\hline \multirow[t]{2}{*}{ Thailand } & $0.018^{* * *}$ & $0.034^{* * *}$ & $0.027^{* * *}$ & $0.017^{* * *}$ & $0.016^{* * *}$ & $0.018^{* * *}$ \\
\hline & & $\begin{array}{c}\text { Using } 10 \% \\
\text { criterion }\end{array}$ & & & $\begin{array}{c}\text { Using } 15 \% \\
\text { criterion }\end{array}$ & \\
\hline Countries & A & $\beta_{\mathrm{L}}$ & $\beta^{\mathrm{U}}$ & A & $\beta_{\mathrm{L}}$ & $\beta^{\mathrm{U}}$ \\
\hline Pakistan & $\mathrm{n} / \mathrm{a}$ & $\mathrm{n} / \mathrm{a}$ & $\mathrm{n} / \mathrm{a}$ & $\mathrm{n} / \mathrm{a}$ & $\mathrm{n} / \mathrm{a}$ & $\mathrm{n} / \mathrm{a}$ \\
\hline Philippine & 0.029 & $0.015^{* * *}$ & $0.051^{* * *}$ & 0.029 & $0.012^{* * *}$ & $0.034^{* * *}$ \\
\hline Thailand & 0.016 & $0.011^{* * *}$ & $0.014^{* * *}$ & 0.015 & $0.009^{* * *}$ & $0.012^{* * *}$ \\
\hline \multicolumn{7}{|c|}{ Panel B: Linear herding estimates for CSAD on market portfolio } \\
\hline & & $\begin{array}{l}\text { Using 1\% } \\
\text { criterion }\end{array}$ & & & $\begin{array}{l}\text { Using 5\% } \\
\text { criterion }\end{array}$ & \\
\hline Countries & A & $\beta_{\mathrm{L}}$ & $\beta^{\mathrm{U}}$ & A & $\beta_{\mathrm{L}}$ & $\beta^{\mathrm{U}}$ \\
\hline Pakistan & $\mathrm{n} / \mathrm{a}$ & $\mathrm{n} / \mathrm{a}$ & $\mathrm{n} / \mathrm{a}$ & $\mathrm{n} / \mathrm{a}$ & $\mathrm{n} / \mathrm{a}$ & $\mathrm{n} / \mathrm{a}$ \\
\hline Philippine & $0.024^{* * *}$ & $0.018^{*}$ & $0.04^{* * *}$ & $0.021^{* * *}$ & $0.015^{* * *}$ & $0.041^{* * *}$ \\
\hline \multirow[t]{2}{*}{ Thailand } & $0.013^{* * *}$ & $0.025^{* * *}$ & $0.02^{* * *}$ & $0.012^{* * *}$ & $0.012^{* * *}$ & $0.013^{* * *}$ \\
\hline & & $\begin{array}{c}\text { Using } 10 \% \\
\text { criterion }\end{array}$ & & & $\begin{array}{c}\text { Using } 15 \% \\
\text { criterion }\end{array}$ & \\
\hline Countries & $\mathrm{A}$ & $\beta_{\mathrm{L}}$ & $\beta^{\mathrm{U}}$ & $\mathrm{A}$ & $\beta_{\mathrm{L}}$ & $\beta^{\mathrm{U}}$ \\
\hline Pakistan & $\mathrm{n} / \mathrm{a}$ & $\mathrm{n} / \mathrm{a}$ & $\mathrm{n} / \mathrm{a}$ & $\mathrm{n} / \mathrm{a}$ & $\mathrm{n} / \mathrm{a}$ & $\mathrm{n} / \mathrm{a}$ \\
\hline Philippine & 0.020 & $0.012^{* * *}$ & $0.033^{* * *}$ & 0.019 & $0.011^{* * *}$ & $0.023^{* * *}$ \\
\hline Thailand & 0.012 & $0.008^{* * *}$ & $0.010^{* * *}$ & 0.011 & $0.006^{* * *}$ & $0.009^{* * *}$ \\
\hline
\end{tabular}




\begin{tabular}{|c|c|c|c|c|c|c|}
\hline \multicolumn{7}{|c|}{ Materials } \\
\hline \multicolumn{7}{|c|}{ Panel A: Linear herding estimates for CSSD on market portfolio } \\
\hline & & $\begin{array}{l}\text { Using 1\% } \\
\text { criterion }\end{array}$ & & & $\begin{array}{l}\text { Using 5\% } \\
\text { criterion }\end{array}$ & \\
\hline Countries & A & $\beta_{L}$ & $\beta^{\mathrm{U}}$ & A & $\beta_{L}$ & $\beta^{\mathrm{U}}$ \\
\hline Pakistan & $0.018^{* * *}$ & $0.023^{* * *}$ & $0.017^{* * *}$ & $0.017^{* * *}$ & $0.011^{* * *}$ & $0.012^{* * *}$ \\
\hline Philippine & $0.029^{* * *}$ & $0.04^{* * *}$ & $0.045^{* * *}$ & $0.027^{* * *}$ & $0.017^{* * *}$ & $0.031^{* * *}$ \\
\hline \multirow[t]{2}{*}{ Thailand } & $0.02^{* * *}$ & $0.085^{* * *}$ & $0.083^{* * *}$ & $0.017^{* * *}$ & $0.047^{* * *}$ & $0.049^{* * *}$ \\
\hline & & $\begin{array}{c}\text { Using } 10 \% \\
\text { criterion }\end{array}$ & & & $\begin{array}{c}\text { Using } 15 \% \\
\text { criterion }\end{array}$ & \\
\hline Countries & A & $\beta_{\mathrm{L}}$ & $\beta^{\mathrm{U}}$ & A & $\beta_{\mathrm{L}}$ & $\beta^{\mathrm{U}}$ \\
\hline Pakistan & 0.016 & $0.009^{* * *}$ & $0.010^{* * *}$ & 0.016 & $0.007^{* * *}$ & $0.008^{* * *}$ \\
\hline Philippine & 0.026 & $0.013^{* * *}$ & $0.025^{* * *}$ & 0.025 & $0.012^{* * *}$ & $0.021^{* * *}$ \\
\hline Thailand & 0.016 & $0.030^{* * *}$ & $0.034^{* * *}$ & 0.015 & $0.059^{* * *}$ & $0.061^{* * *}$ \\
\hline \multicolumn{7}{|c|}{ Panel B: Linear herding estimates for CSAD on market portfolio } \\
\hline & & $\begin{array}{l}\text { Using 1\% } \\
\text { criterion }\end{array}$ & & & $\begin{array}{l}\text { Using } 5 \% \\
\text { criterion }\end{array}$ & \\
\hline Countries & A & $\beta_{\mathrm{L}}$ & $\beta^{\mathrm{U}}$ & A & $\beta_{\mathrm{L}}$ & $\beta^{\mathrm{U}}$ \\
\hline Pakistan & $0.013^{* * *}$ & $0.016^{* * *}$ & $0.011^{* * *}$ & $0.012^{* * *}$ & $0.009^{* * *}$ & $0.009^{* * *}$ \\
\hline Philippine & $0.02^{* * *}$ & $0.029^{* * *}$ & $0.037^{* * *}$ & $0.019^{* * *}$ & $0.014^{* * *}$ & $0.024^{* * *}$ \\
\hline \multirow[t]{2}{*}{ Thailand } & $0.015^{* * *}$ & $0.059^{* * *}$ & $0.061^{* * *}$ & $0.012^{* * *}$ & $0.033^{* * *}$ & $0.035^{* * *}$ \\
\hline & & $\begin{array}{c}\text { Using } 10 \% \\
\text { criterion }\end{array}$ & & & $\begin{array}{c}\text { Using } 15 \% \\
\text { criterion }\end{array}$ & \\
\hline Countries & A & $\beta_{\mathrm{L}}$ & $\beta^{\mathrm{U}}$ & A & $\beta_{\mathrm{L}}$ & $\beta^{\mathrm{U}}$ \\
\hline Pakistan & 0.012 & $0.007^{* * *}$ & $0.008^{* * *}$ & 0.011 & $0.006^{* * *}$ & $0.007^{* * *}$ \\
\hline Philippine & 0.017 & $0.011^{* * *}$ & $0.019^{* * *}$ & 0.016 & $0.010^{* * *}$ & $0.016^{* * *}$ \\
\hline Thailand & 0.011 & $0.021^{* * *}$ & $0.025^{* * *}$ & 0.010 & $0.017^{* * *}$ & $0.019^{* * *}$ \\
\hline \multicolumn{7}{|c|}{${ }^{*}$ Significant at $0.1,{ }^{* *}$ Significant at 0.05 and ${ }^{* * *}$ Significant at 0.01} \\
\hline
\end{tabular}

The table 3 presents the herding estimates from the following equations

Panel A: $\operatorname{CSSD}_{\mathrm{t}}=\alpha+\beta_{1}^{\mathrm{U}} \mathrm{D}_{\mathrm{t}}^{\mathrm{U}}+\beta_{2}^{\mathrm{L}} \mathrm{D}_{\mathrm{t}}^{\mathrm{L}}+\varepsilon_{\mathrm{t}}$

Panel B: $\operatorname{CSAD}_{\mathrm{t}}=\alpha+\beta_{1}^{\mathrm{U}} \mathrm{D}_{\mathrm{t}}^{\mathrm{U}}+\beta_{2}^{\mathrm{L}} \mathrm{D}_{\mathrm{t}}^{\mathrm{L}}+\varepsilon_{\mathrm{t}}$

\subsection{Linear herding estimates}

The results of linear CSSD and CSAD (Table 3) in extreme upper and lower industries movement of Thailand and Philippines report significant positive values for both the coefficient $\beta^{\mathrm{U}}$ and $\beta^{\mathrm{L}}$, which infers the absence of herding and the results 
are consistent with the study of Javed et al. (2011) and Gleason et al. (2004).Herding is only observed in energy sector of Pakistan during the extreme upper and lower industry movements of CSSD and CSAD.

\subsection{Nonlinear herding estimates}

Table 4: Regression Coefficients for Nonlinear Herding Models

\begin{tabular}{|c|c|c|c|c|c|c|}
\hline \multicolumn{7}{|c|}{ Consumer Discretionary } \\
\hline & & $\begin{array}{l}\text { Whole Sam- } \\
\text { ple (CSSD) }\end{array}$ & & & $\begin{array}{l}\text { Whole } \\
\text { Sample } \\
\text { (CSAD) }\end{array}$ & \\
\hline Countries & A & $\gamma_{1}$ & $\gamma_{2}$ & A & $\gamma_{1}$ & $\gamma_{2}$ \\
\hline Pakistan & $0.016^{* * *}$ & $0.796^{* * *}$ & $-9.010^{* * *}$ & $0.011^{* * *}$ & $0.768^{* * *}$ & $-10.689^{* * *}$ \\
\hline Philippine & $0.016^{* * *}$ & $1.560^{* * *}$ & $-3.339^{* * *}$ & $0.010^{* * *}$ & $1.099^{* * *}$ & $-4.256^{* * *}$ \\
\hline Thailand & $0.013^{* * *}$ & $0.542^{* * *}$ & $-1.116^{*}$ & $0.009^{* * *}$ & $0.422^{* * *}$ & $-1.0880^{*}$ \\
\hline \multicolumn{7}{|c|}{ Energy } \\
\hline & & $\begin{array}{l}\text { Whole Sam- } \\
\text { ple (CSSD) }\end{array}$ & & & $\begin{array}{l}\text { Whole } \\
\text { Sample } \\
\text { (CSAD) }\end{array}$ & \\
\hline Countries & A & $\gamma_{1}$ & $\gamma_{2}$ & A & $\gamma_{1}$ & $\gamma_{2}$ \\
\hline Pakistan & $0.0026^{* * *}$ & $0.2089^{* * *}$ & $-3.0213^{* * *}$ & $0.0059^{* * *}$ & $0.5107^{* * *}$ & $-7.2245^{* * *}$ \\
\hline Philippine & $0.0134^{* * *}$ & $1.0435^{* * *}$ & $-4.5597^{* * *}$ & $0.0086^{* * *}$ & $0.9602^{* * *}$ & $-4.277^{* * *}$ \\
\hline Thailand & $0.0113^{* * *}$ & 0.0243 & $9.2278^{* * *}$ & $0.008^{* * *}$ & $0.0867^{* * *}$ & $5.5821^{* * *}$ \\
\hline \multicolumn{7}{|c|}{ Financials } \\
\hline & & $\begin{array}{l}\text { Whole Sam- } \\
\text { ple (CSSD) }\end{array}$ & & & $\begin{array}{l}\text { Whole } \\
\text { Sample } \\
\text { (CSAD) }\end{array}$ & \\
\hline Countries & A & $\gamma_{1}$ & $\gamma_{2}$ & A & $\gamma_{1}$ & $\gamma_{2}$ \\
\hline Pakistan & $0.013^{* * *}$ & $0.785^{* * *}$ & $-10.959^{* * *}$ & $0.009^{* * *}$ & $0.688^{* * *}$ & $-10.303^{* * *}$ \\
\hline Philippine & $0.018^{* * *}$ & $1.343^{* * *}$ & $-10.413^{* * *}$ & $0.01^{* * *}$ & $0.923^{* * *}$ & $-5.751^{* * *}$ \\
\hline Thailand & $0.014^{* * *}$ & $0.459^{* * *}$ & -0.398 & $0.01^{* * *}$ & $0.343^{* * *}$ & -0.266 \\
\hline \multicolumn{7}{|c|}{ Industrials } \\
\hline & & $\begin{array}{l}\text { Whole Sam- } \\
\text { ple (CSSD) }\end{array}$ & & & $\begin{array}{l}\text { Whole } \\
\text { Sample } \\
\text { (CSAD) }\end{array}$ & \\
\hline Countries & A & $\gamma_{1}$ & $\gamma_{2}$ & A & $\gamma_{1}$ & $\gamma_{2}$ \\
\hline Pakistan & $0.01^{* * *}$ & $1.15^{* * *}$ & $-24.895^{* * *}$ & $0.007^{* * *}$ & $0.882^{* * *}$ & $-17.285^{* * *}$ \\
\hline
\end{tabular}




\begin{tabular}{|c|c|c|c|c|c|c|}
\hline Philippine & $0.013^{* * *}$ & $0.944^{* * *}$ & $-5.393^{* * *}$ & $0.009^{* * *}$ & $0.719^{* * *}$ & $-3.809^{* * *}$ \\
\hline Thailand & $0.013^{* * *}$ & $0.605^{* * *}$ & $-1.823^{* * *}$ & $0.009^{* * *}$ & $0.462^{* * *}$ & $-1.118^{* * *}$ \\
\hline \multicolumn{7}{|c|}{ Information Technology } \\
\hline
\end{tabular}

The table 4 presents the herding estimates from the following equations

Panel A: $\operatorname{CSSD}_{\mathrm{t}}=\alpha+\gamma_{1}\left|\mathrm{R}_{\mathrm{it}}\right|+\gamma_{2} \mathrm{R}_{\mathrm{it}}^{2}+\varepsilon_{\mathrm{t}} \& \operatorname{CSAD}_{\mathrm{t}}=\alpha+\gamma_{1}\left|\mathrm{R}_{\mathrm{it}}\right|+\gamma_{2} \mathrm{R}_{\mathrm{it}}^{2}+\varepsilon_{\mathrm{t}}$

The results of nonlinear CSSD and CSAD (table 4) reveal the herding patterns in all sectors. Herding is detected in consumer discretionary, energy, financials and material sector of Pakistan and Philippine. The herding is detected in the industrial sector of all the three countries i.e., Pakistan Philippine and Thailand. Information technology is the only sector that holds the assumption of rational asset pricing model with the positive significant values $\Upsilon_{1}$ and $\Upsilon_{2}$ coefficients. The positive sign shows the level of dispersion increases and negative sign shows the level of dispersion decreases in returns (Christie \& Huang, 1995 and Vo \& Phan (2017). The results are consistent with the study of Chien, Mei and Kuan (2013). In this study herding is observed in Technology and utility sector in emerging markets like China, Korea, and Hong Kong. Another study of Zheng et al. (2017) also uncovers herding in the different sector of Asian stock markets. 
Table 5: Regression Coefficients for Bull and Bear Herding Models

\begin{tabular}{|c|c|c|c|c|c|c|}
\hline \multicolumn{7}{|c|}{ Consumer Discretionary } \\
\hline & & $\begin{array}{c}\text { Up Market } \\
\text { Cond } \\
\left(\mathrm{R}_{\mathrm{m}}>0\right)\end{array}$ & & & $\begin{array}{l}\text { Down Mar- } \\
\text { ket }\left(\mathrm{R}_{\mathrm{m}}<0\right)\end{array}$ & \\
\hline Countries & A & $\gamma_{1}^{\mathrm{UP}}$ & $\gamma_{2}^{\mathrm{UP}}$ & A & $\gamma_{1}^{\mathrm{DW}}$ & $\gamma_{2}^{\mathrm{DW}}$ \\
\hline Pakistan & $0.014^{* * *}$ & $0.438^{* * *}$ & $-4.020 * * *$ & $0.015^{* * *}$ & $0.296^{* * *}$ & -0.068 \\
\hline Philippine & $0.013^{* * *}$ & $0.771^{* * *}$ & $8.513^{* * *}$ & $0.012^{* * *}$ & $0.707^{* * *}$ & -0.022 \\
\hline Thailand & $0.012^{* * *}$ & $0.270^{* * *}$ & $2.620^{* * *}$ & $0.011^{* * *}$ & $0.136^{* * *}$ & $2.446^{* * *}$ \\
\hline \multicolumn{7}{|l|}{ Energy } \\
\hline & & $\begin{array}{c}\text { Up Market } \\
\text { Cond } \\
\left(\mathrm{R}_{\mathrm{m}}>0\right)\end{array}$ & & & $\begin{array}{l}\text { Down Mar- } \\
\text { ket }\left(\mathrm{R}_{\mathrm{m}}<0\right)\end{array}$ & \\
\hline Countries & A & $\gamma_{1}^{\mathrm{UP}}$ & $\gamma_{2}^{\mathrm{UP}}$ & A & $\gamma_{1}^{\mathrm{DW}}$ & $\gamma_{2}^{\mathrm{DW}}$ \\
\hline Pakistan & $0.0081^{* * *}$ & $0.3572^{* * *}$ & $-6.2842^{* * *}$ & $0.0069^{* * *}$ & $0.3255^{* * *}$ & $-2.2153^{*}$ \\
\hline Philippine & $0.0102^{* * *}$ & $0.8799^{* * *}$ & $-2.5685^{* * *}$ & $0.0108^{* * *}$ & $0.7009^{* * *}$ & $-1.5857^{* *}$ \\
\hline Thailand & $0.01^{* * *}$ & $-0.0615^{* *}$ & $9.3572^{* * *}$ & $0.0078^{* * *}$ & $0.1962^{* * *}$ & $0.9794^{* * *}$ \\
\hline \multicolumn{7}{|l|}{ Financials } \\
\hline & & $\begin{array}{c}\text { Up Market } \\
\text { Cond } \\
\left(\mathrm{R}_{\mathrm{m}}>0\right)\end{array}$ & & & $\begin{array}{l}\text { Down Mar- } \\
\text { ket }\left(\mathrm{R}_{\mathrm{m}}<0\right)\end{array}$ & \\
\hline Countries & A & $\gamma_{1}^{\mathrm{UP}}$ & $\gamma_{2}^{\mathrm{UP}}$ & A & $\gamma_{1}^{\mathrm{DW}}$ & $\gamma_{2}^{\mathrm{DW}}$ \\
\hline Pakistan & $0.011^{* * *}$ & $0.452^{* * *}$ & $-6.584^{* * *}$ & $0.01^{* * *}$ & $0.457^{* * *}$ & $-5.256^{* * *}$ \\
\hline Philippine & $0.013^{* * *}$ & $0.762^{* * *}$ & -1.023 & $0.012^{* * *}$ & $0.573^{* * *}$ & -0.522 \\
\hline Thailand & $0.012^{* * *}$ & $0.268^{* * *}$ & 0.929 & $0.012^{* * *}$ & $0.108^{* * *}$ & $2.329^{* * *}$ \\
\hline \multicolumn{7}{|l|}{ Industrials } \\
\hline & & $\begin{array}{c}\text { Up Market } \\
\text { Cond } \\
\left(\mathrm{R}_{\mathrm{m}}>0\right)\end{array}$ & & & $\begin{array}{l}\text { Down Mar- } \\
\text { ket }\left(\mathrm{R}_{\mathrm{m}}<0\right)\end{array}$ & \\
\hline Countries & A & $\gamma_{1}^{\mathrm{UP}}$ & $\gamma_{2}^{\mathrm{UP}}$ & A & $\gamma_{1}^{\mathrm{DW}}$ & $\gamma_{2}^{\mathrm{DW}}$ \\
\hline Pakistan & $0.011^{* * *}$ & $0.53^{*}$ & -11.219 & $0.007^{* * *}$ & $0.783^{* * *}$ & -12.977 \\
\hline Philippine & $0.011^{* * *}$ & $0.556^{* * *}$ & 1.21 & $0.011^{* * *}$ & $0.422^{* * *}$ & -0.279 \\
\hline Thailand & $0.011^{* * *}$ & $0.384^{* * *}$ & $1.232^{* *}$ & $0.011^{* * *}$ & $0.221^{* * *}$ & $1.022^{* * *}$ \\
\hline $\begin{array}{l}\text { Information } \\
\text { Technology }\end{array}$ & & & & & & \\
\hline
\end{tabular}




\begin{tabular}{|c|c|c|c|c|c|c|}
\hline & & $\begin{array}{c}\text { Up Market } \\
\text { Cond } \\
\left(\mathrm{R}_{\mathrm{m}}>0\right)\end{array}$ & & & $\begin{array}{l}\text { Down Mar- } \\
\text { ket }\left(\mathrm{R}_{\mathrm{m}}<0\right)\end{array}$ & \\
\hline Countries & A & $\gamma_{1}^{\mathrm{UP}}$ & $\gamma_{2}^{\mathrm{UP}}$ & A & $\gamma_{1}^{\mathrm{DW}}$ & $\gamma_{2}^{\mathrm{DW}}$ \\
\hline Pakistan & $\mathrm{n} / \mathrm{a}$ & $\mathrm{n} / \mathrm{a}$ & $\mathrm{n} / \mathrm{a}$ & $\mathrm{n} / \mathrm{a}$ & $\mathrm{n} / \mathrm{a}$ & $\mathrm{n} / \mathrm{a}$ \\
\hline Philippine & $0.018^{* * *}$ & 0.647 & 19.379 & $0.015^{* * *}$ & $0.913^{* * *}$ & -6.044 \\
\hline Thailand & $0.011^{* * *}$ & $0.301^{* * *}$ & $6.296^{* * *}$ & $0.011^{* * *}$ & $0.193^{* * *}$ & $3.69^{* * *}$ \\
\hline \multicolumn{7}{|l|}{ Materials } \\
\hline & & $\begin{array}{c}\text { Up Market } \\
\text { Cond } \\
\left(\mathrm{R}_{\mathrm{m}}>0\right)\end{array}$ & & & $\begin{array}{l}\text { Down Mar- } \\
\text { ket }\left(\mathrm{R}_{\mathrm{m}}<0\right)\end{array}$ & \\
\hline Countries & A & $\gamma_{1}^{\mathrm{UP}}$ & $\gamma_{2}^{\mathrm{UP}}$ & A & $\gamma_{1}^{\mathrm{DW}}$ & $\gamma_{2}^{\mathrm{DW}}$ \\
\hline Pakistan & $0.011^{* * *}$ & $0.343^{* * *}$ & $-1.54^{*}$ & $0.012^{* * *}$ & $0.137^{* * *}$ & $3.945^{* *}$ \\
\hline Philippine & $0.015^{* * *}$ & $0.736^{* * *}$ & -0.489 & $0.015^{* * *}$ & $0.444^{* * *}$ & 0.329 \\
\hline Thailand & $0.008^{* * *}$ & $0.788^{* * *}$ & $6.21^{* * *}$ & $0.006^{* * *}$ & $1.035^{* * *}$ & -0.314 \\
\hline \multicolumn{7}{|c|}{${ }^{*}$ Significant at $0.1,{ }^{* *}$ Significant at 0.05 and ${ }^{* * *}$ Significant at 0.01} \\
\hline
\end{tabular}

The table 5 presents the herding estimates from the following equations

Panel A: $\operatorname{CSAD}_{\mathrm{t}}^{\mathrm{UP}}=\alpha+\gamma_{1}^{\mathrm{UP}}\left|\mathrm{R}_{\mathrm{i}, \mathrm{t}}^{\mathrm{UP}}\right|+\gamma_{2}^{\mathrm{UP}}\left(\mathrm{R}_{\mathrm{i}, \mathrm{t}}^{\mathrm{UP}}\right)^{2}+\varepsilon_{\mathrm{t}} \mathrm{R}_{\mathrm{i}, \mathrm{t}}<0 \&$ $\operatorname{CSAD}_{\mathrm{t}}^{\text {DowN }}=\alpha+\gamma_{1}^{\text {Down }}\left|\mathrm{R}_{\mathrm{i}, \mathrm{t}}^{\text {DowN }}\right|+\gamma_{2}^{\text {DowN }}\left(\mathrm{R}_{\mathrm{i}, \mathrm{t}}^{\text {Down }}\right)^{2}+\varepsilon_{\mathrm{t}} \mathrm{R}_{\mathrm{i}, \mathrm{t}}<0$

\subsection{Bull and bear herding estimates}

The results of bull and bear market trends (table 5) also reveal the signs of herding in all sectors as consistent with the findings of Gebka and Wohar (2013). During bullish market trend of Pakistan's consumer discretionary, bullish and bearish market trends of Pakistan's, energy and financial, bullish market trend of Philippine's energy, bullish market trends of Pakistan's health care, bearish market trends of Pakistan's and Thailand's healthcare, bearish market trend of Philippine's telecommunication and utilities sectors exhibit herding but in sectors like industrial, information technology and material investor dose not herd during bullish and bearish market trends. The findings of this research study suggest that firstly, herding is associated with the volatility of industries. There is a direct link between herding and volatility. More the industry is volatile more the herding occurs. Secondly, the herding effect is stronger in similar industries rather than the whole market.

\section{Conclusion}

The study focused on three emerging markets (Pakistan, Philippines and Thailand) to investigate the presence of herding behavior. Different models and theories were 
examined including the "herding" behavior concept linked with investors. Review of the literature reveals that many studies have been conducted for examining the herding behavior. Similarly, this study has mentioned those studies and found that herding exists in markets and investors behave irrationally.

This study took 262 companies of 6 sectors as the study sample for empirical investigation. Secondary data was taken for the period of 2009-2016. Daily returns were considered.

Research methodologies/techniques used were descriptive analysis and return dispersion models. The CSAD and CSSD, non-linearity regression and the bullish and bearish effect of market on herding are the part of our analysis.

Firstly, the study report that there is no sign of herding detected in the linear CSSD model. Secondly, in the linear CSAD model the only sign of herding is observed in Pakistan's energy sector. Furthermore, the non-linearity regression uncovers the herding behavior, all 6 sectors show herding behavior except information technology sector and forth, in the bullish and bearish market movements herding is found in all sectors of emerging countries except industrial, information technology and material sector.

Linear models of return dispersion based on cross-sectional standard deviations (CSSD) and cross-sectional absolute standard deviations (CSAD) does not appropriate to measure the herding pattern. The main findings supported this argument in that the results of the non-linear model revealed a statistically significant negative sign of herding in industries.

From the study's results it is inferred that investors behave rationally until the markets are in normal conditions; however, when there are some "Ups and Downs" in the market, investors deviate from rationality and form the "herd" behavior to keep them aligned with the market. The results are in line with Arjoon and Bhatnagar (2017) showing herding is present in both up and down markets, but is stronger during rising markets. If this herd behavior provides abnormal returns, then the investors appreciate their decision, however, if the herd behavior ends up with loses then the investors blame the market. Further research on herding phenomena can be carried out firstly, by using some other methodologies for example "State Space Model" can be utilized to detect herding. Secondly, further working on the linear model is required to check whether it is appropriate measure or not. Thirdly, the current study which is based on daily returns can be extended to weekly and monthly returns. Implications of this study is that findings of this study can be utilized by managers from various sectors to ascertain their performance and facilitate decision making and and findings 
from this study can help shareholders to analyze the stocks and share prices and also compare this study findings with same sectors in other emerging markets

\section{References}

Arjoon, V., \& Bhatnagar, C. S. (2017). Dynamic herding analysis in a frontier market. Research in International Business and Finance, 42, 496-508.

Asma, M., Mollah, S., \& Keasey, K. (2014). A cross-country analysis of herd behavior in Europe: Journal of International Financial Markets, Institutions and Money, 32, 107-127.

Avery, C., \& Zemsky, P. (1998). Multidimensional uncertainty and herd behavior in financial markets. American economic review, 724-748.

Balcilar, M., Demirer, R., \& Hammoudeh, S. (2013). Investor herds and regime-switching: Evidence from Gulf Arab stock markets. Journal of International Financial Markets, Institutions and Money, 23, 295-321.

Balcılar, M., Demirer, R., \& Ulussever, T. (2017). Does speculation in the oil market drive investor herding in emerging stock markets?. Energy Economics, 65, 50-63.

Bowe, M., \& Domuta, D. (2004). Investor herding during financial crisis: A clinical study of the Jakarta Stock Exchange. Pacific-Basin Finance Journal, 12(4), 387-418.

Balcilar, M., Demirer, R., \& Hammoudeh, S. (2013). Investor herds and regime-switching: Evidence from Gulf Arab stock markets. Journal of International Financial Markets, Institutions and Money, 23, 295-321.

Chien-Chiang Lee, Mei-Ping Chen \& Kuan-Mien Hsieh (2013) Industry herding and market states: evidence from Chinese stock markets, Quantitative Finance, 13:7, 1091-1113, DOI: $10.1080 / 14697688.2012 .740571$

Chiang, T.C., Zheng, D., 2010. An empirical analysis of herd behavior in global stock market. Journal of Banking and Finance 34, 1911-1921.

Christie, W.G. \& Huang, R.D. (1995) Following the Pied Piper: Do Individual Returns Herd around the Market? Financial Analysts Journal, 51, 31-37.https://doi.org/10.2469/faj.v51.n4.1918

Chen, M.-P., \& Hsieh, k.-M. (2013). Industry herding and market states: evidence from Chinese stock markets AU-Lee, Chien-Chiang. Quantitative Finance, 13(7), 1091-1113.

Cipriani,M.,\& Guarino, A. (2008). Transaction costs and informational cascades in financial markets. Journal of Economic Behavior E Organization, 68(3-4), 581-592.

Chang, E.C., Cheng, J.W., Khorana, A., 2000. An examination of herd behavior in equity markets: an international perspective. Journal of Banking and Finance 24 (10), 1651-1699.

Demirer, R., \& Kutan, A. M. (2006). Does herding behavior exist in Chinese stock markets? Journal of International Financial Markets, Institutions and Money, 16(2), 123-142. https://doi.org/10.1016/j. intfin.2005.01.002 
Demirer, R., Kutan, A. M., \& Zhang, H. (2014). Do ADR investors herd? Evidence from advanced and emerging markets. International Review of Economics and Finance, 30, 138-148.

Demirer, R., Kutan, A., \& Chen, C. (2010). Do investors herd in emerging stock markets? Evidence from the Taiwanese market. Journal of Economic Behavior and Organization, 76, 283-295.

Dhar, R., \& Zhu, N. (2006). Up Close and Personal: Investor Sophistication and the Disposition Effect. Management Science,52(5), 726-740.

Dehghani, P. \& Sapian, R. Z. Z., 2014. Sectorial herding behavior in the aftermarket of Malaysian IPOs. Venture Capital: An International Journal of Entrepreneurial Finance, 16(3), pp. 227-246.

Economou, F., Kostakis, A., \& Philippas, N. (2010, June). An examination of herd behavior in four mediterranean stock markets. In European economics and finance society conference paper.

Economou, F., Kostakis, A., \& Philippas, N. (2011). Cross-country effects in herding behaviour: Evidence from four south European markets. Journal of International Financial Markets, Institutions and Money, 21(3), 443-460.

Gleason, K. C., Lee, C. I., \& Mathur, I. (2003). Herding behavior in European futures markets. Finance Letters, 1(1), 5-8.

Gleason, K. C., Mathur, I., \& Peterson, M. A. (2004). Analysis of intraday herding behavior among the sector ETFs. Journal of Empirical Finance, 11(5), 681-694.

Guney, V. Kallinterakis, G. Komba, (2016) Herding in Frontier Markets: Evidence from African Stock Exchanges, Journal of International Financial Markets, Institutions $\mathcal{E}$ Money (2016), doi: http://dx.doi. org/10.1016/j.intfin.2016.11.001

Glbka, B., \& Wohar, M. E. (2013). International herding: Does it differ across sectors? Journal of International Financial Markets, Institutions and Money, 23, 55-84.

Hsieh, M., Yang, T., Yang, Y., \& Lee, J. (2011). Evidence of herding and positive feedback trading for mutual funds in emerging Asian countries. Quantitative Finance, 11(3), 423-435.

Jhandir, S. U., \& Elahi, M. A. (2015). Analysis of Herd Behavior Using Quantile Regression: Evidence from Karachi Stock Exchange (KSE). SSRN Electronic Journal. https://doi.org/10.2139/ssrn.2622413

Javed, T., Zafar, N \& Hafeez, B (2011). Herding behavior in Karachi stock exchange: International Journal of Management Sciences and Business Research, 2(2), 19-28.

Kremer, S., Nautz, D., 2013. Causes and consequences of short-term institutional herding. Journal of Banking and Finance 37, 1676-1686.

Lakshman, M.V., Basu, S., Vaidyanathan, R., 2013. Market-wide herding and the impact of institutional investors in the Indian capital market. Journal of Emerging Market Finance 12, 197-237.

Lin, A. Y., \& Swanson, P. E. (2003). The behavior and performance of foreign investors in emerging 
equity markets: Evidence from Taiwan. International Review of Finance, 4(3-4), 189-210.

Litimi, H., BenSaida, A., \& Bouraoui, O. (2016). Herding and excessive risk in the American stock market: A sectoral analysis. Research in International Business and Finance, 38, 6-21.

Patterson, D. M., \& Sharma, V. (2007). Did Herding Cause the Stock Market Bubble of 1998-2001?. University of Michigan-Dearborn.

Philippas, N., Economou, F., Babalos, V., \& Kostakis, A. (2013). Herding behavior in REITs: Novel tests and the role of financial crisis. International Review of Financial Analysis, 29, 166-174.

Peranginangin, Y., Ali, A.Z., Brockman, P. \& Zurbruegg, R. 2016, 'The impact of foreign trades on emerging market liquidity', Pacific-Basin Finance Journal, vol. 40, pp. 1-16.

Prechter, R.R., 2001. Unconscious Herding Behavior as the Psychological Basis of Financial Market Trends and Patterns. Journal of Psychology and Financial Markets, 2(3), pp.120-125.

Shah, M. U. D., Shah, A., \& Khan, S. U. (2017). Herding behavior in the Pakistan stock

exchange: Some new insights. Research in International Business and Finance, 42, 865-873. https://doi. org/10.1016/j.ribaf.2017.07.022

Simon, H. A. (1955). A behavioral model of rational choice. The quarterly journal of economics, 69(1), 99-118.

Spyrou, Spyros. "Herding in financial markets: a review of the literature." Review of Behavioral Finance 5, no. 2 (2013): 175-194.

Vo, X. V., \& Phan, D. B. A. (2017). Further evidence on the herd behavior in Vietnam stock market. Journal of Behavioral and Experimental Finance, 13, 33-41.

Wermers, R. (1999). Mutual fund herding and the impact on stock prices. the Journal of Finance, 54(2), $581-622$.

Yao, J., Ma, C., \& He, W. P. (2014). Investor herding behavior of Chinese stock market, International Review of Economics and Finance, 29, 12-19.

Youssef, M., \& Mokni, K. (2018). On the effect of herding behavior on dependence structure between stock markets: Evidence from GCC countries. Journal of Behavioral and Experimental Finance, 20, $52-63$.

Zheng, D., Li, H., \& Chiang, T. C. (2017). Herding within industries: Evidence from Asian stock markets. International Review of Economics $\mathcal{E}$ Finance, 51, 487-509. 
